BULLETIN (New Series) OF THE

AMERICAN MATHEMATICAL SOCIETY

Volume 47, Number 1, January 2010, Pages 145-154

S 0273-0979(09)01281-6

Article electronically published on October 15, 2009

\title{
DISCUSSION ON THE EXISTENCE AND UNIQUENESS OR MULTIPLICITY OF SOLUTIONS OF THE AERODYNAMICAL EQUATIONS
}

\author{
CHAIRMAN: DR. J. VON NEUMANN
}

\begin{abstract}
Editor's NOTE. Dr. von Neumann wrote us that his comments were improvised and unsystematic and have retained this character in spite of minor corrections made afterward. For that reason Dr. von Neumann's contribution is rendered as a part of the discussion and not as a separate lecture.
\end{abstract}

\section{Wednesday morning August 17, 1949}

\section{von Neumann:}

I would like to make some remarks about the general hydrodynamical discussion on motions in one dimension, following Riemann's theory, which was expounded by Dr. Burgers and subjected to a critical analysis by Dr. McVittie. The question as to whether a solution which one has found by mathematical reasoning really occurs in nature and whether the existence of several solutions with certain good or bad features can be excluded beforehand, is a quite difficult and ambiguous one. This subject has been considered in the classical literature as well as in the more recent literature, on widely varying levels of rigor and of its opposite. In summa, it is quite difficult ever to be sure of anything in this domain. Mathematically, one is in a continuous state of uncertainty, because the usual theorems of existence and uniqueness of a solution, that one would like to have, have never been demonstrated and are probably not true in their obvious forms.

To this day, the only thing of any degree of generality that we possess is the classical discussion by Riemann, and this very strictly in one dimension and very strictly in the isentropic case. In this case at least, Riemann proved that there are no discontinuities. He also gave the exact conditions under which there can be a solution at all and he proved that in those cases there is only one. So he proved that the number of solutions is either zero or one. He also showed that it is zero in general, i.e., unless certain (infinitely many) very stringent conditions are satisfied. Thus, unless the initial state of the gas fulfills some very particular conditions, the (continuous) solution will cease to exist after some definite finite time. Riemann also inferred, essentially by physical insight, what happens when the continuous solution ceases to exist. He made it very plausible that a discontinuity of a certain type, a "shock wave", develops.

This was subsequently independently rediscovered, and further developed, by Hugoniot. It is also true that in the entire literature up to 1910, i.e., up to the time of the work of Rayleigh and G. I. Taylor, there was a considerable confusion

This article has been reprinted from [1] and contains pp. 75-82 of that text. 
and disagreement between the authors on exactly what the shock looks like. The reason is this: If one assumes the continuity of all relevant parameters in gas theory, and uses the differential equations of this theory (I mean those of compressible, nonviscous, nonconducting media), then all one postulates is the conservation of matter, of momentum and energy. The conservation of entropy then appears as a mathematical consequence. One can paraphrase this by reasoning that the system of mechanics may not conflict with thermodynamics, therefore entropy must not decrease, and since the equations of mechanics are reversible, entropy cannot increase either. Hence it must stay constant and so it is perfectly fair that one gets the conservation of entropy.

This argument sounds convincing, but it is wrong. That this is so can be seen from what happens when shock waves are considered; there the entropy will change, as a mathematical consequence of matter, of momentum and energy. Because of this it is no longer true that every solution of the equations of mechanics is compatible with physics. And it is mathematically, by the way, not true, that for any reasonable statement of the initial conditions and boundary conditions, there will always be one and only one solution. The fact is that if you do not permit discontinuities and demand that the equations of continuum mechanics hold (again for the nonviscous, nonconducting case), i.e., if you exclude discontinuities and apply the usual differential equations, which is what Riemann did, then you find that solutions will not exist in general.

If you permit discontinuities and add to these discontinuities the reasonable conditions of fit (Hugoniot's shock conditions), then you discover the next peculiarity: In every case where trouble arises, there are at least two solutions, namely a shock of the kind that occurs in nature, and the inverse shock, where the discontinuity reflects a pressure decrease instead of an increase, and entropy decreases instead of increasing. You consequently have to add to this mathematical system the further information, which does not come from mechanics, that entropy may change, if it increases, but not otherwise. Thus one concludes that discontinuities must be permitted, but every time discontinuities arise, it is necessary to exclude $50 \%$ of the possibilities. This is a beautiful example of how the reversible system of mechanics can yield a description of an irreversible piece of nature.

This is not a mathematically rigorous statement because the existence and uniqueness of the solutions under these conditions have never been demonstrated. Indeed, I doubt that anybody at this moment has any idea as to how to prove it. As far as I know, even the singularity which describes how a shock develops from a continuous motion has never been correctly discussed, even in one dimension. Furthermore, almost nothing is known about the peculiar singularities which accompany the development of a shock in more than one dimension, except in very special cases.

Thus there exists a wide variety of mathematical possibilities in fluid mechanics, with respect to permitting discontinuities, demanding reasonable thermodynamic behavior, etc., etc. There probably exists a set of conditions under which one and only one solution exists in every reasonably stated problem. However, we have only surmises as to what it is and we have to be guided almost entirely by physical intuition in searching for it. It is therefore impossible to be very specific about any point. And it is difficult to say about any solution which has been derived, with any degree of assurance, that it is the one which must exist in nature. 


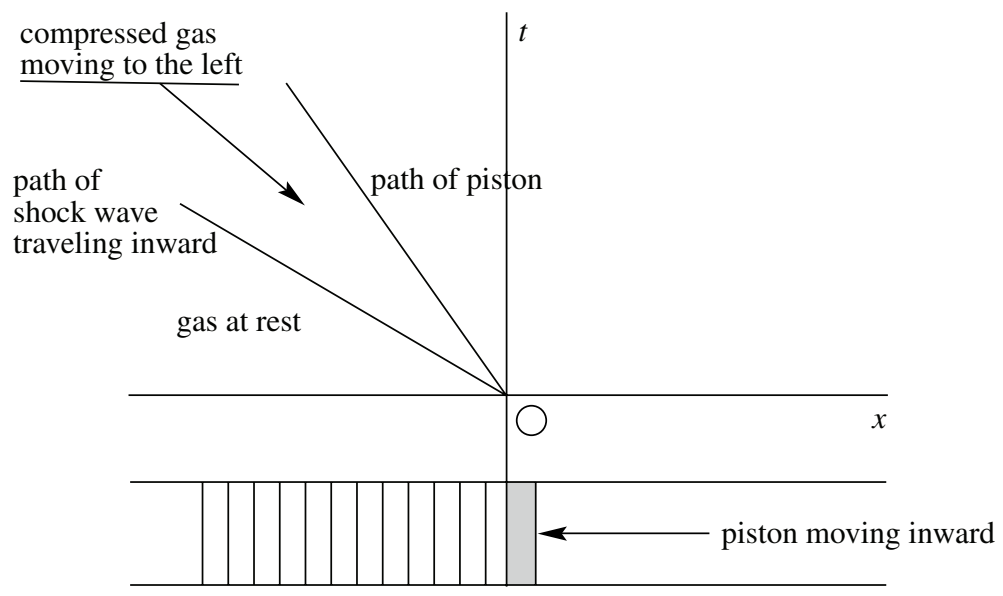

Figure 1

One may now ask to what extent the solutions, which were described by Prof. Burgers are verified and whether one should believe them as a whole? There is a certain amount of quasi-mathematical plausibility for them. There is, I think, a reasonable amount of physical plausibility and there is some physical confirmation in a few critical cases.

Let me describe the situation in a little more detail. Assume that in this diagram (Figure 1) you have a gas occupying part of the $x$-axis, let us say to the left of $x=0$, which should represent a wall. The wall is supposed originally to be at rest and at the instant $t=0$ it begins to move in some way. Then the following is true: Depending on whether the motion is in or out, two completely different things will take place. If you move it in, a discontinuity, a shock wave, will travel inwards, as indicated in Figure 1, where for simplicity a constant velocity of the wall has been assumed. If you start to move it out, another phenomenon takes place (Figure 2): You obtain a system of rarefaction waves of a type which has

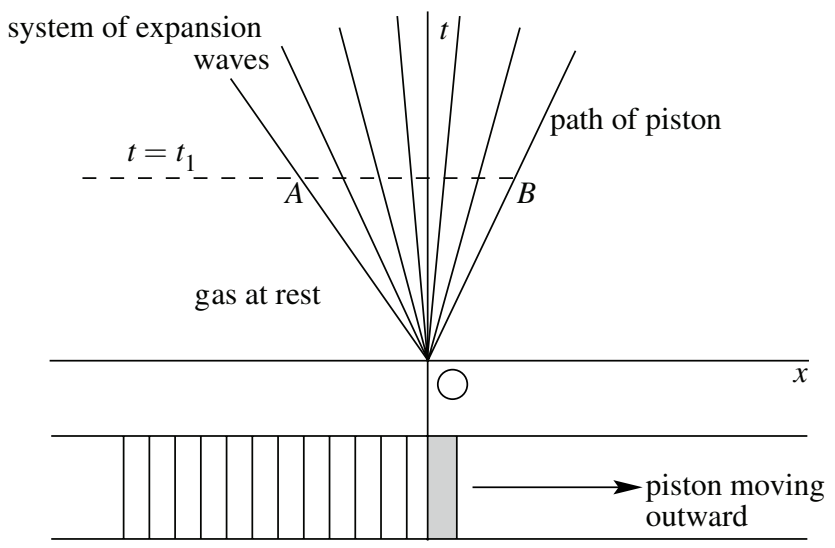

Figure 2 
been described by Riemann, and which afterwards has found its analogue in the socalled Prandtl-Meyer expansion, occurring in cases of stationary supersonic motion in two dimensions. In the system of expansion waves, all hydrodynamical quantities, pressure, density, temperature, and velocity appear to be constant along straight lines in the $x, t$-diagram. When the wall has a constant velocity, all these straight lines start from the origin as pictured in Figure 2. (The same situation is obtained in the case considered by Dr. Burgers in his Figure 2.)

Now it is to be observed that when in the case of expansion you consider the distribution of the pressure at a given instant $t=t_{1}$, you will find constant pressure in a region extending to the point $A$ (in this region the gas is still at rest), thereafter the pressure drops, until we reach the moving wall at $B$. Although in this case there is no discontinuity of the pressure itself, there is at $A$ a discontinuity in $\partial p / \partial x$ (and also in $\partial p / \partial t)$.

With increasing time the profile expands. In the initial state, we have a discontinuity of the pressure itself which immediately afterwards resolves itself; the resolution at first is extremely steep and then gets flatter and flatter.

In the system of expansion waves of Figure 2 there is conservation of entropy; in the shock wave of Figure 1 there is increase of entropy. Mathematically a solution for the case of the outward moving wall can be constructed in which a discontinuity is propagated without resolution. There is no mathematical reason at all why you should not use a solution of that type, but it will lead to a decrease of entropy.

It will be clear that the results which have been derived from the continuum theory can be valid only to the extent to which you can assume that hydrodynamical properties can be defined locally. You certainly cannot do this in the immediate neighborhood of the shock. When the ordinary theory is perfected by taking into consideration viscosity and heat conductivity, the conditions in the neighborhood of the shock can be analyzed to a certain extent, as was shown long ago, first by Rayleigh and G. I. Taylor (1910); their work was amplified by R. Becker (1922) and still further amplified by J. L. Thomas (1943). There is one perfectly plausible result, namely that a few mean free paths away from the shock, things can be described satisfactorily by the phenomenological theory. But in the first few mean free paths, that is, inside the shock region you find out, of course, that there is not a discontinuity at all, but a region where deviations from the classical MaxwellBoltzmann velocity distribution of the kinetic theory of gases occur.

There is a further difficulty in the expansion case considered by Burgers. It was accepted that the front advances into a vacuum. It is evident that you cannot get the normal conditions of kinetic theory here either, because the density of the gas goes to zero at the front, which means that the mean free path of the molecules will go to infinity. This means that if we are in the expanding gas and approach the (theoretical) front, we will necessarily come to regions where the mean free path is larger than the distance from the front. In such regions one cannot use the hydrodynamical equations. But, as in the case of the shock wave, where ordinary conditions are reached at a distance of a few mean free paths from the shock itself, so in the case of expansion into a vacuum, at a short distance from the theoretical front, one comes into regions where the mean free path is considerably smaller than the distance from the front, and where again the classical hydrodynamical equations can be applied. If this is applied to expanding interstellar clouds, I think that in order that the classical theory be true down to $1 / 1000$ of the density of the clouds, 
it is necessary that the distance from the theoretical front should be of the order of a percent of a parsec.

Mathematically one may doubt whether the solution considered here, with Riemann's system of expansion waves, is the only solution. In fact, not only may one doubt it, but it's certainly true that there are other solutions, for instance the negative shock already mentioned before. This negative shock has to be excluded for thermodynamical reasons. But I do not think that anybody at this moment knows whether there may not be some supersophisticated combination of shocks and Riemann expansions and I don't know what other phenomena might go with it.

One must be terribly careful in accepting such extra solutions. Occasionally the simplest hydrodynamical problems have several solutions, some of which are very difficult to exclude on mathematical grounds only. For instance, a very simple hydrodynamical problem is that of the supersonic flow of a gas through a concave corner, which obviously leads to the appearance of a shock wave. In general, there are two different solutions with shock waves, and it is perfectly well known from experimentation that only one of the two, the weaker shock wave, occurs in nature. But I think that all stability arguments to prove that it must be so, are of very dubious quality.

As far as the Riemann expansion is concerned, there is a certain experimental confirmation for it. I do not know whether anybody has ever taken the trouble to measure gas waves with interferometric methods for the case of expansion into a vacuum. But many experiments of a related kind have been made with so-called shock tubes, referring to the case of two gases of equal temperature and different pressures, with a wall between them which is suddenly removed. In this case you get a Riemann expansion in the gas of high density and a shock wave in that of low density (compare Burgers' lecture, Figure 5). The strength of the shock is easy to measure and if this comes out according to the theory, you may assume that the rarefaction will also be according to theory. Such measurements have been made many times. The strength of the shock as a function of the ratio of the pressures on the two sides, has been repeatedly surveyed, very carefully to one part in 50 for a ratio of up to 12 , and to a lesser degree of exactness for ratios up to 1000 . Hence, I think there is a reasonable kind of physical evidence for the correctness of the picture.

In addition there is another situation, which is almost equivalent, namely when phenomena do not take place in one linear dimension and in time, but when they are taking place stationarily in a supersonic flow in two dimensions. In that case flow through a concave corner produces the shock wave and flow around a convex corner gives the Prandtl-Meyer expansion fan. This situation has been investigated most extensively and most wind tunnel designs are based on this and similar cases. A vast amount of quantative information is available, all of which shows that the theory of the Prandtl-Meyer expansion is physically correct.

I want to apologize for having taken up so much of the limited time and I will stop here and would like to ask what other remarks there are.

\section{Liepmann:}

I would like to add a remark about the question of the two shock waves. I think that the experiments cannot be safely cited to settle whether only the solution with the weaker shock appears in nature, because the theoretical case refers to an infinite 
wall (or to the flow along the two sides of an infinite wedge), which case cannot be realized in practice. With the stronger one of the two shock waves you have subsonic flow behind the shock wave, which means that behind the shock wave you have a region where the theory of the elliptic differential equation applies and where the field is influenced by the boundary conditions at a finite or an infinite distance downstream. In the case of the other shock wave the velocity remains supersonic, so that you have conditions such as those obtained with hyperbolic equations. Thus one cannot exclude a priori that conditions downstream may influence the flow and thus may lead to a predilection for one type of shock wave about the other type.

In the case of the Prandtl-Meyer fan, for the flow around a corner, it is true that quite often you get experimentally a combination of shock and expansion waves. The deviations from the theory here are evidently connected with the existence of a boundary layer of the corner walls.

In the question of the discontinuity, it seems to me that as soon as you leave out of the differential equations the terms referring to viscosity and heat conduction, then you have to introduce a new integral relation, which you take from thermodynamics. That is, you have to use the entropy concept. This is necessary since you omit a very large part of the equations. If you do keep viscosity and heat conduction, then you do not need the explicit introduction of entropy: you get the shock solution from a continuous field.

If one considers a body of length $\ell$ moving through a gas at a velocity $U$ it is possible to define a parameter $\operatorname{Re} / M^{2}$ ( $\mathrm{Re}=$ Reynolds number and $M=$ Mach number), which essentially represents the number of molecular collisions in the time $\ell / U$. This similarity parameter can be used to decide over the ranges of applicability of the equations. With $\operatorname{Re} / M^{2} \gg 1$, one can use the equations of nonviscous hydrodynamics; with $\mathrm{Re} / M^{2} \sim 1$, we have viscous and slip hydrodynamics; with $\operatorname{Re} / M^{2} \ll 1$ we come to molecular flow.

\section{von Neumann:}

I would like to answer Dr. Liepmann's remark. In the case of supersonic flow through a concave corner, there are situations where one can produce the other shock (the steeper shock). But in all cases I have seen, one could always argue that the situation was in some sense improperly described. Thus, the boundary conditions far away are often of such a nature that you explicitly exclude one of the two shocks and force the appearance of the other one. Besides, these complicated angular phenomena are probably due - I suppose this was your opinion too - to some boundary effect, not at the actual wall, but at the end of the boundary layer.

\section{Temple:}

I should like to add one further development to a point which you made, Mr. Chairman, when you studied the solutions of the one-dimensional nonsteady motion and those of the two-dimensional steady motion. In the latter case it is well known that a transformation can be carried out and that the two components of the velocity $u$ and $v$ can be taken as new variables, except in certain exceptional circumstances. Those exceptional circumstances are those in which the components $u$ and $v$ are not independent, but are functions of one another. All these exceptional solutions of two-dimensional steady flow are either Prandtl-Meyer expansions, or cases where the fan of straight characteristic lines does not just diverge from one single point, but springs from points on a certain curve. Now if I understand Prof. McVittie's question, one of the main points which he made in his lecture was that the 
solutions to which Prof. Burgers has directed our attention, are in fact the analogue of the exceptional solutions in the case of the two-dimensional steady flow. They are the simplest type of solution, but from a mathematical point of view they are rather exceptional, because they correspond to the fact that a certain determinant becomes zero or infinite.

The cases of the Prandtl-Meyer flow can indeed be realized only when very special precautions are taken to get a uniform field of flow at entry and so forth. The suggestion is that the solution to which Prof. Burgers has directed our attention may also require very special initial conditions. It is therefore necessary to examine whether the astronomical conditions correspond to the initial conditions implicit in Burgers' cases.

\section{von Karman:}

I would like to say something about this question of uniqueness of solutions. I don't think that there is any reason that if you put a problem in a form which has no physical meaning, there shall not be two solutions. And I think the case of stationary motion as such belongs to this category, because it can occur only as a limiting case. Any physical process starts from somewhere and goes to somewhere. In the case of the two shock waves, if instead of considering a stationary motion you consider an accelerated motion, you will first get a detached shock wave ahead of the obstacle (when the Mach number has just passed through unity). Then, with increasing velocity the solution will approach the correct solution for the steady case, I should think, without any difficulty. Such a case comes near to what you can actually realize in an experiment. Is that not correct?

\section{von Neumann:}

I may not have chosen that example which fits best to your argument. It has, of course, to be admitted that to postulate stationarity is to postulate a general trait of the solution one wants, which may hold only approximately in the physical situation that can actually be realized. However, it is not necessary to take the stationary flow through a corner. The following problem also has two solutions. If you take a plane shock which hits a wall and you consider the reflection of the shock from the wall, then under a wide variety of conditions (in fact, in most cases) there are two solutions. In this case stationarity has not been postulated.

\section{von Karman:}

I only mean the following thing. I suppose we start from a certain state of rest of the gas, which must be a solution of our equations. Then we change the conditions gradually and follow the system step by step. I believe that in such a case you will always get a solution and only one solution. There is no proof that there is only one, but I believe it to be so. For, after all, a gas is a molecular system, which follows the general equations of classical mechanics.

But if you take first an infinite cone, or an infinite wedge - both of which are situations which can never be realized - and furthermore you ask for a stationary solution; in such a case there is no reason why there should be only one solution. Since the equations are non-linear, you can often, without violating continuity, pass from one solution to another one by following an envelope, and in such a case you can scarcely find a mathematical reason why one solution should be preferred to the other. But if you start from an actually existing (observed) state and then determine the next phase, I believe you will find only one completely determined result. 
Concerning Dr. von Neumann's example of the reflection of waves from a wall, I do not know the answer, but I believe that no case in which infinitely extending waves or walls are involved is really defined physically.

\section{Heisenberg:}

I have one question in connection with these applications of the hydrodynamical equations. Should one assume from the beginning that these equations actually could be used to such a large extent? If we take the case of the gas expanding into a vacuum, the density at the front is so low that the mean free path becomes larger than the distance to the assumed front. Should one not start from the kinetic picture and say that at the front the molecules will sort themselves out according to their velocities? Then the physical front would be formed by a selection of those molecules which had the highest velocities and did not suffer a collision for a long time. One should expect that there, especially, we have a velocity distribution different from the normal one, and therefore we should not apply the ordinary concepts like temperature and so on. I do not know how big the actual difference is, but I have tried to estimate it. One feels at least that there is a rather large region in which ordinary hydrodynamics cannot be applied, simply because the concepts of temperature and so on would be rather useless.

\section{von Neumann:}

I think that Prof. von Karman also alluded to this problem, when he mentioned the Smoluchowsky region. I suppose he meant that region where you are so close to the surface that the mean free path of the particles is larger than the geometrical dimensions to which you should have to apply your differential equations. Then all the basis for a continuum theory is gone. The only possible course to follow is, I suppose, the same as is followed in star theories, where the atmosphere is discussed with completely different methods from those which are used in the interior. The interior of the sun can be treated in some way like a classical gas, whereas the corona region, of course, is completely different.

Therefore, while it is certainly not rigorously true, don't you think it is sensible, first of all, to apply hydrodynamic theory, and get a solution? If you then discuss in what portions of the field the mean free path is small compared to the distances over which all essential changes occur (one of the most important portions is that where the distance from the boundary is small), it is reasonable to assume that the hydrodynamical equations may at least be used in such regions. When one has to deal with the boundary regions, the Maxwell-Boltzmann theory should be called upon. Now what I have to say is that if one accepts this, and if one estimates how large these extraordinary regions are, in the cases which are of interest in the present context, they turn out to be fairly small. Properly speaking, in the case of the Riemann expansion into vacuum, the region where you have to be careful is quite large but it involves very little substance and very little energy. Hence, in many cases, the correction of the hydrodynamical solution in that region need not be discussed.

\section{Heisenberg:}

I certainly agree chiefly with what you say. I only would like to observe that the failures of hydrodynamical solutions determine the boundary conditions. The boundary conditions react back on the solutions of the hydrodynamic equations, and since these boundary conditions cannot be determined from hydrodynamics and require a detailed study of molecular processes, the two things are interconnected. 


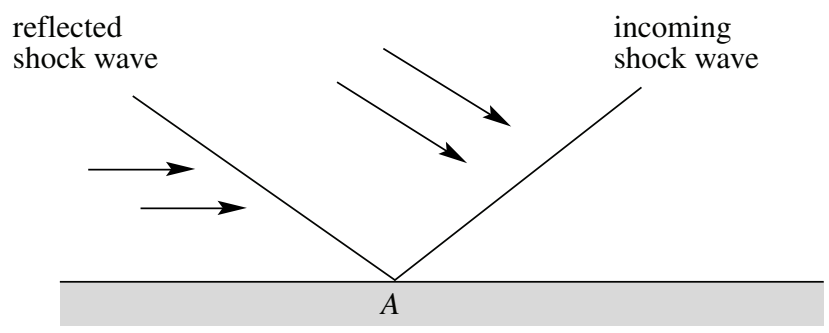

The two shock waves and the point $A$ move together to the right.

FiguRE 3

With you, I believe that on the whole we can talk about hydrodynamical equations and their solutions, but the selection of the solutions to be used depends on the boundary conditions and to this extent we get these non-hydrodynamical parts of the field into our problem.

\section{von Neumann:}

The boundary layer theory for a fluid of low viscosity certainly furnishes a monumental warning. The naive and yet prima facie seemingly reasonable procedure would be to apply the ordinary equations of the ideal fluid and then to expect that viscosity will somehow take care of itself in a narrow region along the wall. We have learned that this procedure may lead to great errors; a complete theory of the boundary layer may give you completely different conditions also for the flow in the bulk of the field. It is possible that the same discipline will be necessary for the boundary with a vacuum. All I would like to say now is that there is yet no evidence for this.

It has been observed what happens if you take air at a pressure of $760 \mathrm{~mm}$ and put it discontinuously into contact with various lower pressures. The observations are fairly precise down to perhaps 10 or $20 \mathrm{~mm}$ and at least qualitatively meaningful down to perhaps $1 \mathrm{~mm}$. The global result is that the expansion conforms to what the naive theory tells. So the indications so far are that the complications of boundary layer theory, which we all know occur when a fluid is in contact with a rigid wall,

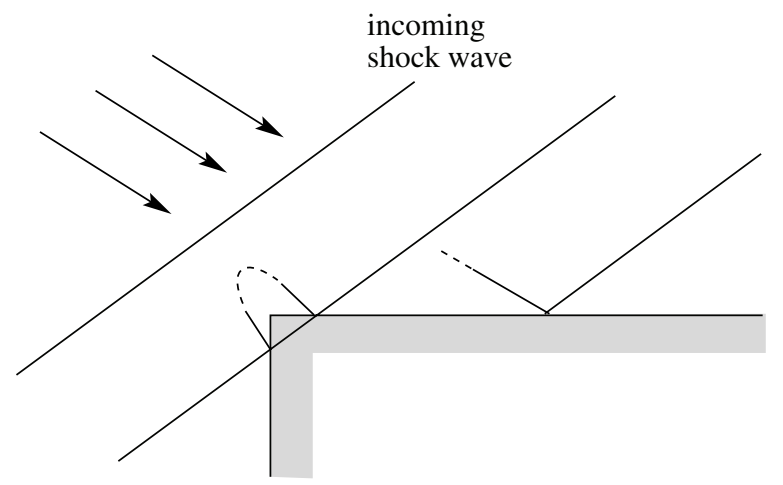

Figure 4 
do not appear to arise when it is in contact with a very low density gas or with a vacuum.

\section{Burgers:}

Dr. von Neumann mentioned a case of nonstationary theory where you have also two solutions: a shock wave hitting a wall. But in the picture you gave (Figure 3) the wall was infinite, so that here again one must ask: How does the situation arise, when you have an actual, finite wall? It may be that you could treat the problem for an actual situation, in which a shock wave travelling in unlimited space reaches the edge of a wall (see Figure 4), you might obtain a definite solution.

\section{von Neumann:}

In that case you assume that the state at the time $t=0$ is given and you ask whether there is or is not a unique continuation of the solution at later times. The answer to this question in its full generality is not known; there seem to be a great many mathematical difficulties.

\section{REFERENCES}

1. John von Neumann, "Discussion on the existence and uniqueness or multiplicity of solutions of the aerodynamical equations", in Problems of Cosmical Aerodynamics. Proceedings of the Symposium on the Motion of Gaseous Masses of Cosmical Dimensions held at Paris, France, August 16-19, 1949, organized by IUTAM and IAU, Chapter 10, pp. 75-85. 